\title{
Clinical Outcome in Human T-Lymphotropic Virus Type 2 Carriers Following Organ Transplantation
}

\author{
Rafael Benito ${ }^{a}$, Joaquina Gil ${ }^{a}$, Juan Sahagún ${ }^{a}$, and Vicente Soriano ${ }^{b, *}$

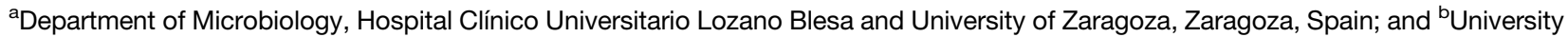 \\ International La Rioja Health Sciences School and Medical Center, Madrid, Spain
}

\begin{abstract}
Background. Frequent and rapid development of myelopathy has been reported in individuals with human T-lymphotropic virus type 1 (HTLV-1) infection following solid organ transplantation. There is no information regarding HTLV-2, a closely related virus that often infects injection drug users.

Methods. This study includes a retrospective analysis of all consecutive organ transplants performed during the last 2 decades at a large reference transplantation unit in Spain. All participants were tested for anti-HTLV antibodies.

Results. A total of 2019 individuals were tested for HTLV during the study period, including 663 potential donors and 1356 recipient candidates. Twelve $(0.59 \%)$ were reactive on initial HTLV serologic screening, but only 6 (all recipients) were confirmed as positive, all for HTLV-2. Two recipients underwent liver transplantation and have remained asymptomatic despite being on tacrolimus for 4 and 8 years, respectively. Likewise, the remaining 4 HTLV-2 carriers have not developed clinical complications potentially associated with HTLV-2.

Conclusions. Unlike HTLV-1 infection, HTLV-2 infection in the transplantation setting does not seem to be associated with rapid development of neurologic complications, Given the cross-seroreactivity between HTLV-1 and HTLV-2, discriminatory rapid tests are urgently needed and would reduce unnecessary organ discharge.
\end{abstract}

$\mathbf{H}$ UMAN T-lymphotropic virus type 1 (HTLV-1) and type $2(\mathrm{HTLV}-2)$ are retroviruses that infect roughly 10 to 15 million people globally. Infection with HTLVs has been associated with the development of hematologic and neurologic conditions, although the evidence for pathogenicity is significantly lower for HTLV-2 than for HTLV-1 [1-3]. Even so, only $10 \%$ of HTLV-1 carriers may develop any of the 2 lifelong characteristic clinical conditions, known as HTLV-associated myelopathy and T-cell leukemia/ lymphoma.

Recent reports have highlighted an increased disease risk in people infected with HTLV-1 following solid organ transplantation [4]. Indeed, rapid development of subacute myelopathy has been reported in more than $40 \%$ of these patients [5,6]. Viral infection may be present in the recipient before transplantation or may have occurred as result of transmission from the infected donor. In either of these situations, the administration of immunosuppressants seems

(C) 2020 The Authors. Published by Elsevier Inc. This is an open access article under the CC BY-NC-ND license (http://

creativecommons.org/licenses/by-nc-nd/4.0/).

230 Park Avenue, New York, NY 10169 to play an important role in driving rapid clinical progression [7]. Based on these observations, HTLV screening has been requested for both donors and recipients before transplantation [8].

Current HTLV serologic screening assays do not distinguish between HTLV-1 and HTLV-2. Collection of this information requires further testing using immunoblot and/ or nucleic acid testing [9]. In the transplantation setting of using cadaveric donors, immediate results of testing are very valuable. Given that no information has been released so far on clinical outcomes for HTLV-2 in the transplant setting,

*Address correspondence to Dr Vicente Soriano, University International La Rioja Health Sciences School and Medical Center, calle Almansa 101, Madrid 28040, Spain. Tel: +34 659687981. E-mail: vicente.soriano@unir.net

$0041-1345 / 20$ https://doi.org/10.1016/j.transproceed.2020.08.029 
Table 1. Main Features of the HTLV Population in Spain

\begin{tabular}{lccc}
\hline & HTLV-1 & HTLV-2 & $P$ Value \\
\hline No. & 389 & 807 & n.s. \\
Median age in years (range) & $41(3-78)$ & $35(14-71)$ & n.s. \\
Male sex & $135(34.7 \%)$ & $613(76 \%)$ & $<.001$ \\
Country of origin: Spain, Latin America, Africa & $65(16.7 \%), 254(65.3 \%), 43(11 \%)$ & $730(90.4 \%), 21(2.6 \%), 14(1.7 \%)$ & $<.001$ \\
Major route of infection: Heterosexual, men having sex & $124(31.5 \%), 6(1.5 \%), 12(3 \%)$ & $32(3.9 \%), 0,609(75.5 \%)$ & $<.001$ \\
$\quad$ with men, injection drug use & $24(6.2 \%)$ & $668(82.8 \%)$ & 1,0 \\
HIV co-infection & $45(11.6 \%), 27(6.9 \%)$ & $<.001$ \\
$\begin{array}{l}\text { Major clinical manifestations: Myelopathy, T-cell } \\
\quad \text { lymphoma }\end{array}$ & & $<.001$
\end{tabular}

Abbreviations: HIV, human immunodeficiency virus; HTLV-1, human T-lymphotropic virus type 1; HTLV-2, human T-lymphotropic virus type 2; n.s., non significant.

we reviewed the last 20 years of our hospital liver transplantation registry.

\section{METHODS}

We conducted a retrospective analysis of all consecutive organ transplants performed during the last 2 decades at 1 large reference transplantation unit in Spain. All were tested for anti-HTLV antibodies.

Over time, HTLV antibody screening was performed using 3 methods: indirect enzyme immunoassay (Abbott, Madrid, Spain), sandwich enzyme immunoassay (Abbott-Murex, Madrid, Spain), and, more recently, double sandwich chemiluminescent microparticle immune assay (Architect, Abbott). All initially HTLVseroreactive samples were further tested using confirmatory/ discriminatory Western blot (Genelabs Technologies Inc., Redwood City, Calif, United States), line immunoassay (Innogenetics N.V., Ghent, Belgium), and/or immunoblot (MP Diagnostics, Copenhagen, Denmark). During the last decade, specific and discriminatory polymerase chain reaction tests were also used.

\section{RESULTS}

In Spain, a national case registry has existed for these 2 viral infections since 1989. To date, 389 individuals with HTLV-1 and 807 with HTLV-2 have been reported nationwide [10]. Table 1 records the main features of these populations. Of note, whereas $66 \%$ of HTLV- 1 carriers are immigrants from Latin America, nearly 90\% of HTLV-2-positive patients are native Spaniards who frequently acknowledged injection drug use.

A total of 2019 patients were tested for HTLV during the study period, including 663 potential donors and 1356 recipient candidates. Twelve patients $(0.59 \%)$ were reactive on initial HTLV serologic screening. All were native Spaniards, and 11 were potential transplantation recipients and 1 was a donor. After discharge from the transplantation procedure path, only 6 recipients were confirmed as positive using confirmatory assays and all had HTLV-2 infection. The remaining patient had either indeterminate or negative results on repeated testing. Of note, the only donor initially HTLV-seroreactive was not confirmed as infected.

Two of 6 potential recipients with HTLV-2 underwent liver transplantation 8 and 4 years ago, respectively. The latter patient was also positive for human immunodeficiency virus. To date, none of them has developed either neurologic or hematologic complications potentially associated with HTLV-2 infection despite continuous immunosuppression with tacrolimus. Likewise, none of the remaining 4 HTLV-2 recipient candidates has developed myelopathy or lymphomas that could be related to HTLV-2.

\section{DISCUSSION}

During the last 2 decades, 2019 individuals have been tested for HTLV, of which 663 were potential donors and 1356 were recipient candidates. Twelve $(0.59 \%)$ were reactive on initial HTLV serologic screening, but only 6 (all recipients) were confirmed as positive, all for HTLV-2. Two underwent liver transplantation and have remained asymptomatic despite being on tacrolimus for 4 and 8 years, respectively. Likewise, the remaining 4 HTLV-2 carriers have not developed clinical complications potentially associated to HTLV-2.

Our results suggest that concerns for rapid and frequent disease development in the transplantation setting should be restricted to HTLV-1 and does not seem to apply to HTLV-2. Therefore, the advent of rapid screening diagnostic tests specific for HTLV-1 must be considered a priority as it would reduce unnecessary organ discharge due to unspecific HTLV-seroreactivity in the transplantation setting.

\section{ACKNOWLEDGMENTS}

The authors are grateful to the Spanish HTLV Network.

\section{REFERENCES}

[1] Araujo A, Hall W. Human T-lymphotropic virus type II and neurological disease. Ann Neurol 2004;56:10-9.

[2] Soriano V, Gutiérrez M, Bravo R, Diaz F, Olivan J, González-Lahoz J. Severe myopathy in an injection drug user coinfected with HIV-1 and HTLV-2. Clin Infect Dis 1994;19:350-1.

[3] Toro C, Blanco F, García-Gascó P, Sheldon J, Benito JM, Rallon NI, et al. HTLV-associated myelopathy/tropical spastic paraparesis in an HIV-positive patient coinfected with HTLV-2 following initiation of antiretroviral therapy. Clin Infect Dis 2007;45:e118-20.

[4] Taylor G. Human T-lymphotropic virus type 1 infection and solid organ transplantation. Rev Med Virol 2018;28:1-23. 
[5] Yamauchi J, Yamano Y, Yuzawa K. Risk of human T-cell leukemia virus type 1 infection in kidney transplantation. N Engl J Med 2019;380:296-8.

[6] Toro C, Rodés B, Poveda E, Soriano V. Rapid development of subacute myelopathy in three organ transplant recipients after transmission of human T-cell lymphotropic virus type 1 from a single donor. Transplantation 2003;75:102-4.

[7] Roc L, de Mendoza C, Fernández-Alonso M, Reina G, Soriano V. Spanish HTLV Network. Rapid subacute myelopathy following kidney transplantation from HTLV-1 donors: role of immunosuppresors and failure of antiretrovirals. Ther Adv Infect Dis 2019;6:2049936119868028

[8] Gallo R, Willems L, Hasegawa H. Global Virus Network's Task Force on HTLV-1. Screening transplant donors for HTLV-1 and -2. Blood 2016;128:3029-31.

[9] Cassar O, Gessain A. Serological and molecular methods to study epidemiological aspects of human T-cell lymphotropic virus type 1 infection. Methods Mol Biol 2017;1582:3-24.

[10] de Mendoza C, Soriano V. HTLV-1 infection is rising up in Spain whereas HTLV-2 is vanishing. AIDS Rev 2019;21:233-5. 